\title{
Lorentz's Transformations and Gravitation in the Granular Space Theory
}

\author{
Vladimir Konushko \\ Protvino, Moscow, Russia \\ E-mail: konushko@mail.ru \\ Received October 19, 2010; revised March 10, 2011; accepted April 12, 2011
}

\begin{abstract}
Research into the read structure of space at ways leads to the conclusion on the existence of a privileged (absolute) system of reference, with all the equations remaining invariant about Lorentz's transformations. The expansion of these transformations makes it possible to obtain easily the Schwarzshild matrix and, also, all the results of Einstein's theory of gravity. The untangling of the physical meaning of velocity as a measure of relative deformation of elementary space cells eliminates, at last, all the paradoxes of Lorentz's transformations and allows visual observation of the mechanism of gravity and Coulomb interaction in imaginary experiments.
\end{abstract}

Keywords: Lorentz's Transformations

\section{Absolute Space Reality}

The radical change in our ideas of space and time expressed in Lorentz's transformations has a deep effect on the whole physics. This fact makes every subsequent generation of researchers reanalyze the unusual ratios following from these transformations since there are a lot of paradoxes here. The results of modern physics force us again to discuss such basic concepts as reality, space and time.

Here we want to understand something real and the meaning of this reality rather than just to manipulate formulas and predict correctly the results of experiment.

When formulating his laws of mechanics Newton introduced the concept of absolute space that always remains the same and static. It was relative to this space that he determined acceleration of bodies. This acceleration was absolute by nature and inertia is considered with reference to this absolute space.

If a gyroscope is placed on the Earth so that it can freely rotate around three mutually perpendicular axes, it retains the directions of the rotating axis with respect to the inertial coordinate system. Therefore, the gyroscope seems to be moving relative to the rotating Earth. Thus, the rotating axis of a free gyroscope is always directed to one and the same star (the proper motion of the star can be neglected due to its huge remoteness).

This example makes us consider again the nature of inertia effects. If the Earth were covered with clouds all the time, we would certainly try to explain the "strange" motion of the gyroscope about the Earth involving Newton's idea about the existence of absolute space to the reference of which the Earth rotates. Indeed, any attempt to find a visible cause of gyroscope axis motion would be vain.

According to Newton, space exists even when it is free of all physical bodies; the effect of inertia will exist in this case as well. It is Newton's opinion that absolute space is only able to act on bodies (to resist to their acceleration) but matter cannot act on this space. Later, criticizing this Newton's theory Einstein said that this declaration contradicts our scientific understanding of effects: how can we visualize something that can exhibit an effect but cannot be acted on?

In 1902 Puancaret in his book "Science and Hypothesis" wrote that "... there is no absolute space and we only get to know relative motions." When studying this book Einstein said in his first description of the Universe model in 1917 that " $\ldots$ in the successive relativity theory there cannot be any inertia about "space" but just mass inertia about each other."

Even materialist-philosophers, however, interpreted the concept of matter as an indestructible and unreadable basis of the entire existing matter.

Later Einstein studied this problem again and considered the case of two bodies infinitely removed and so not 
exhibiting a gravitational effect of ball-shaped bodies which rotate relative to the axis passing through the centre of one of them.

Actually and in Einstein's theory of gravity the rotation of a body is absolute motion which was first noted by Weyl.

The introduction of this hypothesis (abstraction) as absolute space turned out to be very fruitful, and only 350 year later we can reanimate this assumption by stating that it is not only space but also all the bodies in the Universe are made up of only one element of a tree-dimensional elementary cell the size of which was determined by Planck in 1900 [1].

If we accept this assumption, we get a privileged system of reference - absolute. In practice its role is played well enough by system of reference related to far-removed star called the system of "state" stars whose proper motion can be neglected because of their huge remoteness.

\section{Equality of Rights of Inertial Systems}

At present all the inertial systems of reference are considered to be physically equivalent and none of them can be preferred to the others. So, inertial systems are equivalent in two respects:

1) Equivalent about the form-invariance of natural laws.

2) Equivalent about the course of physical processes.

From the second statement it follows that any of two inertial systems can be considered static. If the stationary observer wished he would say to the moving one, "Your watch is slow." But the moving observer could say equally well, "My watch is right but yours is slow" because, from his point of view, he is motionless and the so-called stationary observer is moving with the same velocity in the opposite direction.

There is no question that the physical equations in each individual system of reference are equal but the second statement that any inertial system can be referred to as stationary is doubtful.

Let us consider a specific case when two trains, A and $\mathrm{B}$, move to meat each other with the same velocity towards the station where the observer $\mathrm{C}$ is standing. The observers $\mathrm{A}$ and $\mathrm{B}$ begin moving from the same distance to the station $\mathrm{C}$, all of them having high-precision (atomic) watches previously synchronized.

The observer A can consider himself at rest, then it will seem to him that the observer B is moving to meet him with the velocity $2 v(v \ll c)$ and all the physical processes for the observer B must slow down with the factor $\gamma$ :

$$
\gamma=\sqrt{1-\frac{4 v^{2}}{c^{2}}} .
$$

The observer B, in his turn, can consider himself motionless, and all the processes will slow down in the train A with the same factor $\gamma$. When the trains meet, all the three observers register the time by their watches and then compare the results. To their surprise A and B will find that they are absolutely wrong because they have made two mistakes.

1) Their watches will show the same time.

2) The readings of their watches are slower than that of $C$ not by the factor $\gamma$ but by $\gamma^{\prime}$ :

$$
\gamma^{\prime}=\sqrt{1-\frac{v^{2}}{c^{2}}} .
$$

Right was the observer $\mathrm{C}$ who moved slower about the absolute reference system (fixed stars). To escape errors all the three observers were to take only the reference system $\mathrm{C}$, connected with the platform, as a static one. This imaginary experiment can be easily made real but the result of this experiment is quite clear, we have just set it forth.

For over half a century physicists have been observing the deceleration of a physical process in a fast flying elementary particle, a muon, - an increase in lifetime. The observer in the reference system of the flying muon will think that he and the muon are at rest whereas the Earth flies at a huge rate to the opposite direction. Hence, from their standpoint, the rest muons on the Earth move very fast with the Earth, and their lifetime increases as compared to the muons in their system. This conclusion is invalidated completely by the semi-centennial observations. No matter how we manipulate reference systems, a fast-moving muon will always live longer than a static one. So, a real situation cannot be equalized with an imaginary one. Space itself "knows" which muon flies faster about fixed stars.

Finally, let us analyze the clock or twin paradox that has been written about a lot but everybody avoided finding the right solution of this paradox. There was a wish to retain the reciprocity: an inertial system where the twin-astronaut is at rest and the Earth flies away to the opposite direction at the same velocity with the astronaut is believed no worse than a system where the Earth is at rest and the astronaut is moving. As this takes place, the imaginary reference system has equal rights with the real one.

Reciprocity is needed for Lorentz's transformations to form a group. Once Einstein said that «mathematics is the most perfect way of pulling your own nose». It can be just a maidservant of physics but never the queen.

It is stated that a twin-astronaut undergoes acceleration 
and deceleration several times, that is why his physical processes are slowed down to a greater extent. The fact that a twin-traveler may fly away from the Earth, with the same velocity, over a distance of one thousand or one million kilometers is ignored is this case. In the second case the deceleration will be one thousand tines larger than in the first case even though the time of acceleration and deceleration is the same. As a result, the solution of this paradox sounds like a spell: the role of the acceleration undergone by a twin-astronaut is that at the turning point be passes into another noninertial reference system and from this point a different time reading begins.

It is well known that, as physics was developed, all the great achievements brought prejudices and myths which were sometimes helpful and sometimes very harmful for further progress of science. History of science knows a lot of really wonderful ideas which have stood mankind and science in good stead but, nevertheless, have left the stage of science since have not turned out to be real objectively. For example, using the representation of "heatgen" scientists have deduced formulas for thermodynamics not rejected even today.

Absolute space ascertains, finally, a hierarchy of inertial reference systems - another natural law which is not worse at all than the law of conservation of energy or momentum: all physical processes are decelerated to a greater extent in the inertial system moving at a higher velocity about the absolute system of reference.

Only one hundred years after the birth of the specific theory of relativity the granular space theory solves the twin paradox in the most natural way, without stretches and speculations. The reference system in which the twin 0 ' flies into space and the twin 0 stays on the Earth is real. The Earth moves with a lower velocity about stationary stars, and so we consider it static; and since the twin 0 ' flies in his rocket with a high velocity about stationary stars, all his physical processes, including biological ones, are decelerated.

When the twin 0 ' in the rocket thinks that he is at vest about the stars and the Earth flies away in the opposite direction from him, this system is not real but imaginary and unrelated to the existing situation: no matter what he thinks, his rocket flies faster than the Earth and the processes are slowed down or him as before. Thus, neither acceleration nor the inclusion of gravitational affects (Einstein's equivalence principle) are related to this paradox. To solve this paradox we have to go beyond the existing paradigm and to accept absolute material space de facto.

It should be said that Einstein did not like rather a poor word "relativity" introduced in physics by Planck and continued to refer to specific theory of relativity as theory of invariants. Of interest is the following phenome- non: such things can be observed by as for a hundred years but, nevertheless, ignored due to prejudices about what is essential and what is not. The mathematic requirement of reciprocity and, hence, the existence of the elegant Lorentz's group has exceeded the physical part of natural phenomena.

The direct Lorentz's transformation can be expressed as:

$$
\begin{gathered}
x=\frac{x^{\prime}+v t^{\prime}}{\sqrt{l-(v / c)^{2}}}, \\
t=\frac{t^{\prime}+\left(v / c^{2}\right) x^{\prime}}{\sqrt{l-(v / c)^{2}}},
\end{gathered}
$$

where the primed quantities belong to the moving reference system. It is easy to get the inverse Lorentz's transformation:

$$
\begin{aligned}
& x^{\prime}=\frac{x-v t}{\sqrt{l-(v / c)^{2}}}, \\
& t^{\prime}=\frac{t-\left(v / c^{2}\right) x}{\sqrt{l-(v / c)^{2}}} .
\end{aligned}
$$

And this fact was a source of a mistake. Indeed, the physical situation has not change at all: the primed reference system $K^{\prime}$ moves, as before, faster than the system $K$ about fixed stars and the physical processes are slowed down to a greater extent in this system. Starting from 1905 , however, it has been stated that the $K$ ' system can be considered at rest and the $K$ system in this case will move with the velocity $v$ in the opposite direction. This free handling with mathematic symbols has given birth to many paradoxes in Lorentz's transformations and distorted their physical meaning.

It is often written that a moving clock is decelerated but this is in conflict with the principle of relativity. The clock speed in all the inertial reference systems remains constant. They register the physical time similarly in their inertial reference systems. It is not the clock speed that changes but the time of the physical process. The durations of local physical processes measured by a clock in a certain inertial reference system and in any other system are different which again proves the absence of process-invariance.

At present there is even not a hint at why all physical processes are retarded in fast reference systems as well as in gravitational fields. Nobody has ever observed acceleration of processes. It is very surprising to see that the formula incorporating the deceleration of physical processes is the same for fast reference systems and for gravitational fields. 
In case of a gravitational field formed by the mass $M$ the relation between the invariant intrinsic time $\tau$ and the coordinate time $t$ can be expressed thus

$$
(\mathrm{d} \tau)=\left(1-\frac{r_{g}}{r}\right) \cdot(\mathrm{d} t)^{2}=\left(1-\frac{2 G M}{c^{2} r}\right) \cdot(\mathrm{d} t)^{2}
$$

and near the surface of $M$

$$
(\mathrm{d} \tau)^{2}=\left(1-\frac{v_{2}^{2}}{c^{2}}\right) \cdot(\mathrm{d} t)^{2}
$$

where $r_{g}$ is the mass gravitational radius, $v_{2}$ is the escape velocity.

In a similar manner, if particle flies with the velocity $v$ about a motionless reference system, its life time $t$ increases according to the formula

$$
\tau^{2}=\left(1-\frac{v_{2}^{2}}{c^{2}}\right) \cdot t^{2}
$$

where $\tau$ is the life time of a particle in the intrinsic reference system. The fact that these two formulas are absolutely similar shows again that velocity describes both dynamics and statics. In other words, $v^{2} / c^{2}$ is the relative deformation of space cells.

The kinetic energy of a moving particle carries a mass: $E=m c^{2}$. This mass deforms the cells near the particle thus hampering its decay.

The gravitational field in the granular space theory constitutes deformed space cells acting on the particle in quite a similar manner, that is, they hinder its decay thus increasing its life time. That is why we have never observed acceleration of particle decay but only deceleration. And again we can draw the conclusion that all physical and biological processes can be reduced to one: and that is deformation of elementary space cells.

We can say that an essential element of progress in science is the evidence of invalidity of either a theory, on the whole, or some its theses. Despite the fact that all the physical laws in inertial systems have one and the same form, their behavior in most cases is different. The irony is that in 1905 Einstein in his article "Electrodynamics of moving media" studied, maybe, the only case when both the situations conform to reciprocity-the Faraday experiment on initiation of electromagnetic induction.

Let as have a coil with conducting wire around it and connected with a galvanometer and a magnet.

The first situation: the coil is at rest, the magnet is moving - a current is induced in the coil.

The second situation: the magnet is at rest, the coil is moving with the same velocity - the same current is induced in the coil; the coil is moving in the opposite direction.

As applied to the twin paradox, the second situation could mean that we slowed down "by hand" the rocket to the speed of the Earth and imparted the speed of the rocket to the Earth, but in the apposite direction.

Thus, after considering a real experiment complying with reciprocity Einstein drew a wrong conclusion (a typical example of a logical error) regarding the complete equality of inertial systems which caused the absolute reference system and absolute space to be negated and delayed the study into the real material structure of space for a hundred years.

There is another apparent paradox. Let a body fly with the velocity $u=-3 / 4 c$ about an inertial reference frame, and another body flies with the same velocity $v=3 / 4 c$ to meet it. According to the classical law of composition of velocities, the bodies can met with $3 / 2 c>c$. But Lorentz's transformations give a different law of composition of velocities

$$
u^{\prime}=\frac{u-v}{1-u v / c^{2}}=\frac{24 c}{25}<c(!)
$$

Both imaginary and real experiments show that the approach velocity of two bodies is $3 / 2 c$ but this conclusion does not contradict Lorentz's transformations. In this case we can observe the underwater part of velocity: $v^{2} / c^{2}$ is the relative deformation of space cells. In Lorentz's deformations $u$ and $v$ conceal the degree of deformation of elementary cells which cannot be higher than the maximum deformation relating to $c$ [2].

One of the most difficult and unusual Einstein's statements is that the velocity of light is constant in any inertial system independent of the method of its measurement. The sequence of this postulate is the contraction of length of the moving object. Since a body moving in field-free space is not acted upon by any forces, this contraction is called "kinematic". So, this term conceals our unability to understand the essence of this contraction.

To reveal this mystery, let's consider the following imaginary experiment that can be easily become real.

Let an observer A stand on a platform and B in a fast moving train. When the back wall of the carriage comes up with the observer A, the lamp fixed on this wall flares up and the photons begin to travel towards the front wall. Both the observers fix the time of the flash and the moment when the photons reach the front wall. Besides, the observer A on the platform measures the distance L covered by a photon in the time $\Delta T$.

The observer $\mathrm{B}$ in the carriage measures the photon path, too, which equals the carriage length 1 and its travel time $\Delta t$.

Then both the observers calculate the velocity of light in their inertial systems:

and 


$$
u=l / \Delta t=c .
$$

From the standpoint of A the observer B is wrong because he has measured wrongly the photon travel path equal to 1 and, hence, the velocity of light measured by B must be different. Everything became clear when the observers compared the readings of their watches:

$$
\Delta t=\Delta T \sqrt{1-v^{2} / c^{2}}
$$

where $v$ is the carriage speed. But in this case the relation

$$
l=L \sqrt{1-v^{2} / c^{2}}
$$

holds true.

Thus, there is neither real nor "kinematic" contraction in length of objects; this "contraction" means that the observers in different reference systems measure different lengths.

In the second part of this experiment both the observers measure the velocity of light in the case when the photons move from the front wall to the rear, i.e. opposite to the train. All the physical processes will be retarded, as before, near the observer $B$, and the distance covered by the photons is equal to $l$. But the time of movement of the photons from the front wall to the rear one is shorter than $t$ and, hence, the measured velocity of light $C_{2}$ is higher than in the first case: $C_{2}>C_{1}$.

The perfect accuracy of atomic clock at present is sufficient to detect the difference in the velocity of light being measured. The importance of this experiment cannot be overestimated for the following reason. Starting from Galileo, for the following reason. Starting from Galileo, 400 years ago, it has been believed that when being inside an inertial system and not looking out of it we cannot determine whether we move or remain stationary which, in its turn, causes absolute space and absolute reference system to be negated. As it has been noted, absolute space eliminates equality and sets the hierarchy of inertial systems.

Both our imaginary experiment and a future real one, however, fully invalidate this opinion and provide support for the existence of absolute space.

This conclusion could have drawn before by analyzing Sagnac experiment. Michelson experiment, however, averages the velocity of light in different directions and has nothing to do with our consideration.

It is worth noting that both Poincare and Einstein did not accept the existence of absolute space.

The Sagnac effect has been used so far to prove that we can defect the motion of a no inertial frame of reference without leaving it. The importance of this effect, however, is much greater. The essence of this experiment consists in the following. One light beam moves towards the mirrors in a rotating frame of reference and another beam moves to catch up with the mirrors. The velocity of light measured for these beams is different: $C_{1} \neq C_{2}$. This situation is a replica of the above experiment where the velocity of light was measured in two directions in inertial systems.

Does all this mean a downfall of Einstein's hypothesis for steadiness of the velocity of light in inertial systems? The granular space theory gives a "negative" answer to this question since it is the velocity of light that is hides responsible for the maximum deformation of space cells in any inertial reference system.

It should be also noted that for the observer B the objects of the observer A will be elongated (seeming) rather than contracted. This enables as to understand the physical meaning of Schwarzschield's solution.

\section{Physical Meaning of Velocity and Acceleration}

Present-day physics does not reveal the real physical meaning of velocity, so the question arises. If absolute space exits, how must we explain the fact that uniform motion in absolute space does not cause any observable effect while acceleration does?

Our studies just into three physical effects of many other have enabled us to reveal the following natural law: absolute space establishes a hierarchy of inertial reference systems; this law is a manifestation of the velocity of uniform motion.

There is quite a number of physical situations when velocity characterizes rest but not motion. Several phenomena of the kind, paradoxical at first sight, are considered by Feynman in his lectures in Physics [3].

Let us consider the instance when the electric charge and the magnet are at rest. Let a point charge be at rest near the center of a magnetic bar (Figure 1).

Everything is at rest, so that energy doesn't change with time either and are constant. But Poynting's vector show the presence of energy fluxes here because is not zero. If we observe the energy flux we can be sure that it circulates around this system.

The energy, however, remains constant: all that enters

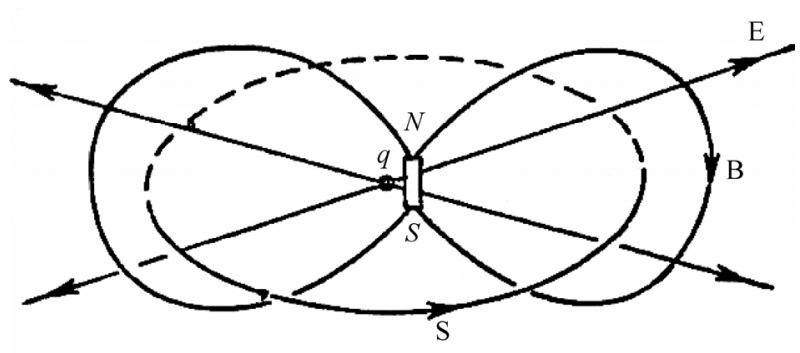

Figure 1. The charge and the magnet result is Poynting's vector circulating along a closed loop. 
this volume flows out of it again. This resembles the circular flow of incompressible water. So, in this seemingly static situation we observe an energy flux which looks rather absurd!

To our regret we can observe that in this case energy "travels around a circle" but, as we know, the energy and momentum fluxes are proportional to each other, so here we have momentum circulation. But momentum circulation means the presence of a momentum. Hence, a stationary field possesses a momentum. This enigmatic circulating energy flux, which seems at first something incomprehensible, is in fact absolutely necessary. But there is also a real momentum flux.

The following situation is not of less interest. Imagine two electrons whose velocities are perpendicular so that their paths intersect but, nevertheless, the electrons do not collide. At a certain moment their relative position will be as shown in Figure 2.

The charge $q_{2}$ is only acted upon by an electric force since on its way $q_{1}$ does not set up a magnetic field. But, besides an electric field, it is also a magnetic field that acts on $q_{1}$, so that it moves in the magnetic field set up by the charge $q_{2}$.

The forces acting on these particles do not balance each other, so the action and the counteraction are not equal and the full momentum of substance must change; it does not remain constant. But in this situation the field momentum changes, too. The momentum present by Poynting's vector is not constant. But the variation of particle momentum is exactly compensated for by field momentum, so the total momentum of the particles and the field is retained all the same.

And here a stationary field acquires a momentum, the velocity characterizes rest again.

Spin is a key property of matter, though still imperfectly understood. Initially spin appeared in physics as an intrinsic moment of momentum $M_{e}$ of electron: $M_{e}=\hbar / 2$. Planck's constant has the following dimension:

$$
\hbar=m \cdot v \cdot r \text {. }
$$

Since inside an electron there are no clusters (quarks) consisting of deformed cells, that is, no inner structure it has been so fare believed to be extremely small:

$r_{e}<10^{-17} \mathrm{~cm}$. But the size of any elementary particle is its fundamental characteristic and must be determined only by world constants and rest mass:

$$
r \geq \lambda=\frac{\hbar}{m c} .
$$

Assuming that the size of a particle is negligible, as it has been believed so far, the spinning electron hypothesis offered by Kronig was rejected by both Pauli and Geisenberg with Lorentz as it would have suggested that matter moves with a velocity much higher than that of

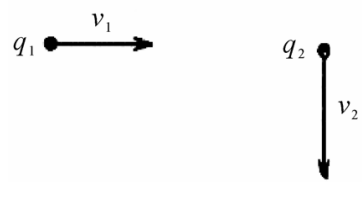

(a)

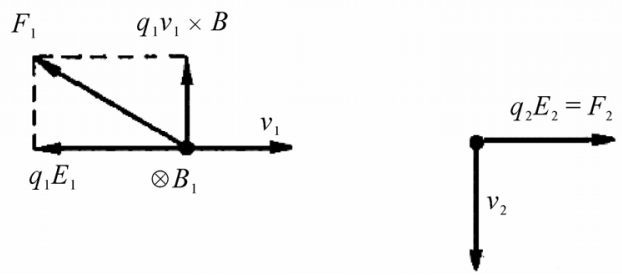

(b)

Figure 2. The forces between two moving charges are not always equal and opposite. "Action" is not equal to "counteraction”.

light.

The granular space theory makes us accept that the size of any elementary particle cannot be less than its Compton wavelength.

Nevertheless, this discovery cannot solve the spin problem: with this size an electron must rotate with the velocity of light $c$ which can be rejected at once. The velocity of light enters into the well-known mass- energy ratio $E=m c^{2}$ which characterizes a body at rest.

We could give more instances of physical effects where the velocity $v$ is characteristic of statics rather than dynamics. This intriguing conclusion changes our concept of the physical meaning of velocity $v$ : the velocity $v$ is on iceberg, its peak being usual travel speed of an object about the fixed count body. The underwater deeper part of the iceberg is related to the deformation of elementary space cells. More specified is the following statement: the quantity $\varepsilon=v^{2} / c^{2}$ denotes the relative deformation of space cells.

This statement enables us to observe almost visually the particle spin: the quantity $c$ in the spin formula (1) characterizes not the speed of electron rotation but the relative deformation of the cells making up the "body" of particle-spiral or torsional deformation-whereas radial cell deformation denotes gravity and electric charge.

The following model gives a rough idea of spin structure. Let us place domino pieces along a circle at intervals equal to the length of one piece and then drop one of them. The falling pieces form a peculiar alignment symbolizing torsional deformation. Similarly, a moving charged particle form around itself the same spiral deformation of space cells designated by us as the magnetic field. Only now we can understand from where space gets spiral properties (Figure 3). 


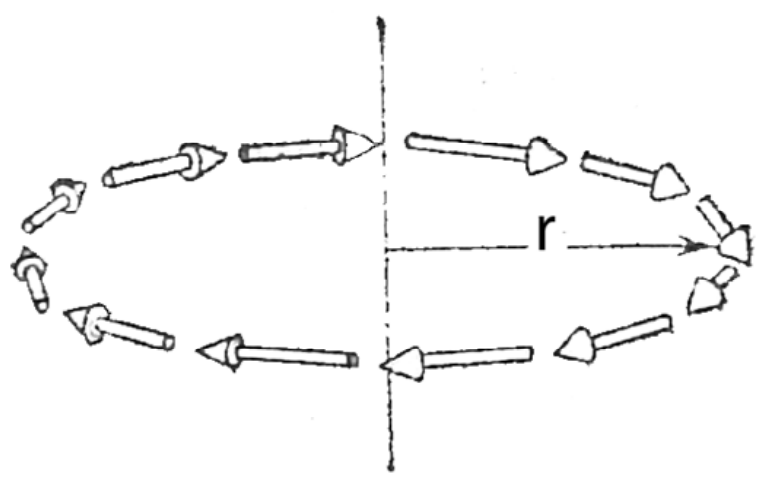

Figure 3. Model of torsional deformation of elementary space cells.

In another work we shall consider Geisenberg inequalities and stability of hydrogen atom. Bohr's attempt to solve this problem involving Geinsenberg inequalities is rejected by experiment, and only a new concept of velocity, momentum, kinetic and potential energy can solve the problem of hydrogen atom stability.

The theory of granule space enables us to observe almost visually not only the velocity $v$-deformation of elementary space cells but also the acceleration $a$-the gradient of this deformation. In its turn, this statement allows understanding the principle of equivalency of an accelerated reference system and a uniform gravity field for all physical processes which represents the fundamental principle of Einstein's theory of gravity. In more detail this subject will be discussed in a separate article.

It is well known that a new theory raises more new questions than it can answer old ones. The key questions of a new theory are related to radial and axial deformation of elementary space cells as well as their clusterization. And the only way of unraveling these secrets is guessing. Even new, when we have not got an accelerator with $E \approx 10^{19} \mathrm{GeV}$, we must mentally increase an electron to the size of a football and try to guess its surface structure since this simplest elementary particle is the key to a huge number of mysteries of the Universe.

Without unraveling the electron structure we shall never be able to solve either the problem of elementary particle mass spectrum nor the boundary line separating animated nature from unanimated.

In our previous works we set forth in detail the concept of granular space and here we are going to recall some foundations of this theory for coherency [4].

The granular structure of space was first mentioned by ancient philosophers, and then, in 1900, Planck found the size of this cell:

$$
L^{*}=\sqrt{\frac{2 G \hbar}{c^{3}}} \approx 1.6 \cdot 10^{-33} \mathrm{~cm} .
$$

In 1972 Beckenstein introduced inexplicitly the ele- mentary cell area $\left(L^{*}\right)^{2} \approx 10^{-66} \mathrm{~cm}^{2}$ and in 1975 the author extended this series by postulating the minimum volume of elementary cell $\left(L^{*}\right)^{3}$, and the whole Universe turns out to be composed only of one element - a cell. According to Wheeller, "space is made up of cells of this size on its deepest level" [5]. At present more and more physicists believe that space has a granular structure and specifies an absolute system of reference [6,7].

In the theory being developed by us a cell is material, three-dimensional and flexible; excessive specification of its characteristics at the first stage of development of the theory may only lead to rough mistakes. All elementary particles are part of space as Klifford foresaw. The formation of a particle demands an additional amount of matter called mass; this mass deforms the inner cells of the particle providing their confinement and, in case of stable particles, produces their stable surface. The "body" of the particle is made up of space cells. It is evident that the rest mass of the particle is invariant because this invariance does not depend on the reference system we use to observe it. But how is the additional matter (mass) arranged in space which is all filled with cells? The most natural thing that can occur is that part of the cells will be pushed out into the area of space external about the particle surface and the cells inside the particle will be deformed. It is obvious that the larger is the mass spent to produce a particle, the stronger is the deformation of inner and outer cells which results in a direct mass-size relationship: the larger the mass, the smaller the size of the particle. Quantitatively this is expressed by the formula for Compton wave length of the particle:

$$
\lambda=\frac{\hbar}{m c}
$$

Consequently, contrary to the existing public opinion, the electron is the largest particle in size-it is the lightest particle if the neutrino is equal to zero.

\section{Has Neutrino Get a Rest Mass?}

Present-day experiments aimed at a search for neutrino oscillations at the same time point out to the existence of a rest mass in an electron neutrino, $m_{v}<1 \mathrm{eV}$. In this case the size of this particle $r_{v}=\hbar / m_{v} c \sim 10^{-5} \mathrm{~cm}$ which is a thousand times larger than the atom size. This is absolutely impossible because as such a particle moves in a medium, the atoms of the medium will pass unimpeded through the particle without interacting with it and without destructing it.

The second cause of absence of a rest mass in a neutrino is the following. As known, neither an electron nor a muon have neutral partners. Hence, the electric charge 
structure (clusters on the particle surface) is a necessary element for forming a stable surface of these particles. As the neutrino has no electric charge, space cannot form its closed shell; the spin alone is unable to do it.

These two causes completely denounce the hypothesis of massive neutrinos and spare experimentalists farther searches for a rest mass in a neutrino. But the oscillating character of motion of both a photon and a mass-free neutrino cannot prohibit neutrino oscillations. The same oscillatory motion of all elementary particles discussed in our article "Weak Interaction and the Nature of Virtual Particles" supports the existence of $\mathrm{K}^{\circ}$ - and $\mathrm{B}^{\circ}$-meson oscillations.

In this case it is very important to note that this mutual transformation of the neutrino $\mathrm{K}^{\circ}$ - and $\mathrm{B}^{\circ}$-meson is caused by the interaction between the non-stationary surface of the particles that "breathes" and the pulsating matter carried by kinetic energy.

The different degree of compression of this matter serves as "a trigger" that makes, for instance, the $\mathrm{K}^{\circ}$ meson decay into two or three particles; it also transfers one type of neutrino into another one.

We have every ground to suppose that it is just this interaction that is responsible for disturbing CP- and T-symmetry.

As part of the particles are forced out into outer space, the external layers of cells are overloaded with matter and their radial and axial (torsion) deformation is taken by other particles as various types of physical fields and a particle spin.

It is evident that the excessive matter made by forced-out cells is equal to the mass of the resultant elementary particle - this peculiar Archimedes effect in microphysics does not depend on whether a particle has either an electric or a strong charge, or a spin. It is just this excessive matter arising so naturally as a result of particle birth that is called by us as potential energy. The intensity of physical interactions depends on which part of this excessive matter can be transferred by space to the interacting particles, and real space hasn't got any other infinite energy.

The deformation of the cells inside a particle may give birth to clusters, that is, bunches of deformed cells known as quarks, and the deformed cells between quarks cause as to introduce intermediate (exchange) particles referred to as gluons. Consequently, neither quarks nor gluons can exist in free state. Outer cells from a layer structure, the former planes in this case are deformed to piece-smooth curved surfaces which gives grounds to introduce an extremely conditional curvature of space. We cannot have a look at our real three-dimensional space from "outside" since there is not the fourth spatial measurement. We can observe the deformation of cell layers by increasing mentally the cell size.

Now let us consider another type of energy-kinetic. Kinetic energy means the amount of substance (matter) transferred by a particle in a given reference system. This matter is arranged asymmetrically: it is running ahead of the particle.

The absence of bond energy between space cells makes it possible to conserve inertia which means that space has zero viscosity.

In the non relativistic limit the kinetic energy:

$$
E=\frac{m_{0} v^{2}}{2}=\frac{1}{2} m_{0} c^{2} \cdot\left(\frac{v^{2}}{c^{2}}\right) .
$$

The coefficient $v^{2} / c^{2}$ shows which part of matter (mass) spent to form the particle itself is constituted by the additional substance moving ahead of the particle. It is this substance that form new particles as two corpuscles collide.

The result of annihilation of electron and positron is that the inner cells making up the bodies of these particles are left in place, the particles give back the space what they once borrowed to build their "bodies" and their mass is spent to form the "bodies" of two photons.

Lomonosov used to say that nature «does not like to luxuriate excesses of things».

In our article "Weak Interaction and Nature of Virtual Particles" we show that the motion of a particle is oscillating by nature and the maximum size of propagation of cell deformation is referred to as the wavelength $\lambda$. The wavelike motion of the matter running ahead of the particle was responsible for the formation of a «centaur», that is, a particle-wave, in its turn, has culminated in numerous misunderstandings in considering quantum phenomena. An electron, for example, has quite a definite size and its spread is out of the question.

This new look on the space structure made as forget about the "centaur" and introduce a new object: "a rider on a horse".

"Horse" means the substance (the mass), carried by kinetic energy moving ahead of the particle, the «rider» the particle itself.

A periodic contraction and then a spread of cell deformation to the size of wavelength enables considering the wave phase. The fact that in reality it is difficult to observe experimentally this complicated motion is one of the sources of probability in quantum theory.

The principle of calibrated symmetry is the principle of relativity in charge space. It was first applied by Fock in 1926 when he could show that the Klein-Fock-Gordon equation for a particle in an electromagnetic field is invariant about the simultaneous phase transformation of the $\psi$-function and gradient transformation of electromagnetic field potential: 


$$
\begin{gathered}
\psi \rightarrow \psi(x) e^{i e f(x)}, \\
A_{v}(x) \rightarrow A_{v}(x)+\partial_{v} f(x) .
\end{gathered}
$$

This extremely morbid situation with probability and indeterminacy will be considered in more detail in the next article.

The rough model of physical field suggested by Maxwell did not comply with reality, and this fact negatively affected the studies into the real space structure. There was a false idea that an electromagnetic wave did not need a carrier and it could freely propagate in empty space. But we are astonished by the sagacity of Faraday and Maxwell who in electric and magnetic lines could see physical reality. As a result, they concluded that electric and magnetic energies are embedded not in the bodies, their source, but in the space around these bodies. This fact gave birth to the concept of a physical field that carries an energy propagating from point to point with a finite velocity. The theory of granular space is a specific realization and materialization of the ideas of these great investigators of Nature. The idea that kinetic energy transfers to mass, and voice verse, mass transforms to kinetic energy is badly perceived in present-day literature. In actual fact, both mass and kinetic and potential energies mean the amount of substance (matter) used to form elementary cells but this matter is distributed in different regions of space.

Any inertial system, be it an elementary particle or a rocket moving about fixed stars has a kinetic energy and, hence, the space cells around it are overloaded with substance and lose flexibility which explains the deceleration of the processes in this inertial system; the higher is the velocity, the stronger is the deceleration.

\section{Spatial Structure and Gravity Effects}

When developing his gravitation theory Einstein used, on the one hand, the discovery connected with Galileo which says that all bodies fall equally fast and, on the other hand, the equality of heavy and inert masses. It is evident that there existed a fundamental relation between the dynamic acceleration of the body dependent on inert mass and the gravitational acceleration conditioned by a heavy mass.

On the basis that an accelerated reference system and a uniform gravitational field are equal for mechanical processes Einstein wrote that his conclusions will be fundament only when this equality is valid for all physical processes or, in other words, if physical laws remain valid with the accelerated reference system replaced by a gravitational field. In this case, when we study theoretically the effects occurring about a uniformly accelerated coordinate system, we can visualize the course of effect in a uniform gravitational field.

When formulating this principle being a version of the principle of equivalence Einstein mode use of the following imaginary experiment.

Imagine an observer $\mathrm{A}$ in a light-tight box far removed from gravitational masses. Now impart uniform acceleration along a direct line to the box. The observer in this case is to perform a number of physical experiments inside the box.

The second observer B is in a similar box optically isolated from outside but placed in a uniform gravitational field, on the surface of the Earth for example. The observer B performs in his experiments similar to the ones carried out by A.

The principle of equivalence says that, if the boxes are not too big and the experiments are made for a short time, all the physical processes will equally in both the boxes and the observers optically isolated from outside are unable to find out if their boxes are in a gravitational field or move with equal acceleration.

And now let us try to understand how, knowing Lorentz's transformations and using the principle of equivalence, we can describe the influence of a gravitational field on physical processes.

Let a non inertial reference system I' move at the moment relative $t_{0}$ an inertial reference system I. Knowing the acceleration of I' about I we can at each instant of time determine the velocity of I' about I. And knowing the velocity we can with the help of Lorentz's transformations relate the coordinates and the time of any event in the system I to the coordinates and the time of the same event in the system I'. It turns out that knowing Lorentz's transformations we can establish the relation between the coordinates and the time in different reference systems but also between other quantities, forces, for example. In other words, if we can describe the physical effects in I, we can describe them in I' using Lorentz's transformations. But the acceleration of I' about $I$ is equivalent, as we know, to the existence of a gravitational field in I'. Consequently, it is possible to calculate the effect of a gravitational field on the course of the physical processes under consideration and answer, for instance, the question: how the gravitational field of the Sun influences the shift of Mercury's perigelium.

At present more and more physicists understand that Lorentz's transformations are applicable not only for inertial reference systems but also in non inertial (accelerated) ones $[8,9]$.

\section{Schwarzschield's Solution}

One of the first great discoveries, after Einstein created 
his theory of gravity, was Schwarzschield's solution of space-time geometry around a point mass. Let us assume, too, that space-time has Minkovsky's geometry at a very long distance from a point mass. So, there is such a radial coordinate $r$ that at a very long distance from the point mass the distance $\mathrm{d} l$ between two close point on the same radius is equal to the difference $\mathrm{d} r$ of their radial coordinates $r_{2}-r_{1}$.

Besides, there exists such a time coordinate $t$ that at a long distance from the point mass the time interval $\mathrm{d} t$ is equal to the difference $t_{2}-t_{1}$.

Let a system connected with the Sun be a fixed reference system and the reference start be placed on the Sun. Since any planet moves faster than the Sun about "fixed stars", two factor should be taken into account in the presence of the linear element $\mathrm{d} s$ : the deceleration of all the physical processes on the planet and the "kinematic" increase in length of sections from the viewpoint of the observer on the planet.

Let us define the way of measuring the rod length from the viewpoint of two observers. Let $l_{0}$ denote the rod length in the fixed reference system connected with the Sun and $l$ be the length of the same rod measured by an observer on a planet, the Mercury for example.

Let the ends of this $\operatorname{rod} X_{1}$ and $X_{2}$ be fixed at the same time instant by the observer in the fixed reference system:

$$
T_{1}=T_{2}
$$

This allows us to reduce the interval $S_{12}^{2}$ in the fixed system only to the spatial portion:

$$
S_{12}^{2}=-\left(X_{2}-X_{1}\right)^{2}=-l_{0}^{2} \text {. }
$$

The some interval in the moving reference system is:

$$
S_{12}^{2}=c^{2}\left(T_{2}^{\prime}-T_{1}^{\prime}\right)-\left(X_{2}^{\prime}-X_{1}^{\prime}\right)^{2} .
$$

But, according to Lorentz's transformations, we have:

$$
T_{2}-T_{1}=\gamma\left[\left(T_{2}^{\prime}-T_{1}^{\prime}\right)-\frac{v}{c^{2}}\left(X_{2}^{\prime}-X_{1}^{\prime}\right)\right] .
$$

Substitution of this value into (2) gives:

$$
S_{2}^{12}=-l^{2}\left(1-\frac{v^{2}}{c^{2}}\right) \text {. }
$$

By comparing (1) and (3) we get;

$$
l=\frac{l_{0}}{\sqrt{1-\frac{v^{2}}{c^{2}}}} .
$$

This purely "kinematic" effect is unrelated, of course, to the real length of the rod; its physical mechanism is considered above.
All the present-day monographs dealing with Lorentz's transformations give another method for measuring the rod length. And again a rod with its length $l_{0}$ is at rest in the Sun's system. But now the ends of the rod $X_{1}^{\prime}$ and $X_{2}^{\prime}$ are fixed at the some time instant by the observer in the moving reference system:

$$
T_{1}^{\prime}=T_{2}^{\prime} \text {. }
$$

Replication of the above-given calculations gives quite the opposite result:

$$
l=l_{0} \sqrt{1-\frac{v^{2}}{c^{2}}}
$$

which means a decrease in rod length.

These results indicate again that there exists an absolute reference system and, in spite of the from-invariance of physical laws relative to Lorentz's transformations, all physical processes, even in inertial systems, come about in different ways, that is, there is no process of invariance.

Correlation of the principles of classical and relativistic cosmology must contain a structure of absolute space as a general system of reference for classical and relativistic description of the Universe.

Below, when considering the motion of planets around the Sun, we can get Schwarzschield's metric without using such a cumbersome body of mathematics as tensor calculus only on condition that the rods fixed in the system of the Sun elongate if they are measured by an observer on a planet.

In the reference system the interval has the form:

$$
\mathrm{d} S^{2}=c^{2} \mathrm{~d} t^{2}-r^{2}\left(\sin ^{2} \theta \mathrm{d} \varphi^{2}+\mathrm{d} \theta^{2}\right)-\mathrm{d} r^{2}
$$

Taking into account two above-mentioned effects taking place in a moving reference system (the system of a planet) we can transform the expression for interval in spherical coordinates:

$$
\begin{aligned}
\mathrm{d} S^{2}= & \left(\sqrt{\left(1-\frac{v^{2}}{c^{2}}\right)}\right)^{2} c^{2} \mathrm{~d} t^{2}-r^{2}\left(\sin ^{2} \theta \mathrm{d} \varphi^{2}+\mathrm{d} \theta^{2}\right) \\
& -\frac{\mathrm{d} r^{2}}{\left(\sqrt{1-\frac{v^{2}}{c^{2}}}\right)^{2}}
\end{aligned}
$$

When writing this element we are guided by the fact that Lorentz's transformations are applicable not only to inertial reference systems but also to non inertial (accelerated) ones.

In regular Lorentz's transformations the quantity $v$ means the velocity of motion of a planet around the Sun. Using the interval (4) we can calculate four gravitational effects once studied by Einstein. To our surprise, all the 
results turn out to be half as much as the experimental ones. Here we could stop trying to use Lorentz's transformations in describing gravitational effects but the appearance of "two" natural numbers, puts us on our guard. In one of our articles we cited E.Kronecker's words that "natural numbers are created by God and all the rest is man's handiwork".

The doublet structure of hydrogen atom was marked by the birth of spin, a unique characteristic of elementary particles. We also know well Tomas's half and Einstein's and de Haas's experiments where a "two" was present, too.

The gravitational field near a massive body is characterized by two velocities: orbital $-v_{1}$ and escape $-v_{2}$. It is $v_{2}$ that is responsible for the real intensity of gravitational field since the object needs this velocity to escape a massive body. The relation between these velocities is very important for our considerations:

$$
v_{2}^{2}=2 v_{1}^{2} .
$$

According to granular space theory, $\varepsilon=v^{2} / c^{2}$ denotes the relative deformation of elementary space cells and velocity characterizes not only motion but also rest. This problem was considered in detail by us in one of our articles "Week Interaction and the Nature of Virtual Particles" [10].

This discovery enables us to introduce generalized Lorentz's transformations where the velocity $v$ entering the well-known root $\sqrt{1-v^{2} / c^{2}}$ must denote a maximum value characterizing this physical effect (situation).

The motion speed of a planet as it revolves around the Sun is an analogue of the orbital velocity, but the gravitational field of the Sun near the planet is characterized by a velocity the square of which is twice as much-an analogue of the escape velocity. And then the physical meaning of the well-known root changes considerably:

$$
\sqrt{1-\frac{v_{1}^{2}}{c^{2}}} \Rightarrow \sqrt{1-\frac{v_{2}^{2}}{c^{2}}}=\sqrt{1-\frac{2 v_{1}^{2}}{c^{2}}},
$$

where $v_{1}$ means the motion speed of a planet along an orbit.

Furthermore:

$$
\sqrt{1-\frac{2 v_{1}^{2}}{c^{2}}}=\sqrt{1-\frac{2 G M_{\oplus}}{r c^{2}}}=\sqrt{1-\frac{r_{g}}{r}},
$$

where $r_{g}=2 G M_{\oplus} / c^{2}$ is the gravitational radius of the Sun first introduced by Schwarzschield in 1916.

Finally, for outer space the following expression is valid:

$$
\mathrm{d} s^{2}=\left(1-\frac{r_{g}}{r}\right) c^{2} \mathrm{~d} t^{2}-r^{2}\left(\sin ^{2} \theta \mathrm{d} \varphi^{2}+\mathrm{d} \theta^{2}\right)-\frac{\mathrm{d} r^{2}}{\left(1-\frac{r_{g}}{r}\right)}
$$

This expression for interval was first received by Schwarzschield in 1916.

In reality a theory can be developed both on a simple and clear basis and (by getting equivalent results) on quite an opposite one. Experience shows, however, that elaborating a theory on the basis of a minimum number of independent axioms is the simplest and the most truthful way.

The essence of Freedman's works consists in the idea that the Universe evolution dynamics results in the inequality $H \neq 0$ ( $H$ is the Hubble constant), that is, the Universe must be an unstationary object.

But there is a twist of fate here, too: though the first model of Universe expansion was built on the basis of Einstein's theory of gravity, the basic conclusions can be drawn within Newton's theory of gravity.

From the viewpoint of history, this slightly paradoxical phenomenon was demonstrated by the English astrophysicists Miln and Maccry in 1934, more than 10 years after Freedman's works were published. And only 90 years after Schwarzschield's works were published, we studied a material granular structure obtained the same results, though much simpler, without using the cumbersome body of mathematics of Riemannian geometry.

Many fundamental physical relations have an extremely simple form. One may say that a very important physical form is certain to have a simple form no matter how complicated its deduction is (the beauty of a physical formula consists, in essence, in this fact).

Eventually, the simplicity of a formula means a high degree of understanding any phenomenon, so high that it allows constructing its very simple physical model.

Bohr told that when you don't understand a physics phenomenon, you write a lot of formula, and when you understand at last you have one or two formula left.

Einstein was not the first to be amazed by a strange similarity between gravitational and inertial phenomena. What is this statement based on? Our conclusion is not less striking than Einstein's assumption: both the acceleration $a$ and the velocity $v$ are another characteristic of deformation of elementary space cells. And if the velocity $v$ characterizes uniform deformation of cells, the acceleration $a$ accounts for the deformation gradient. Consequently, acceleration as well as velocity are characteristic of not only dynamics - motion, but also statics - an object at rest or a physical field.

A lift moving with the acceleration $g$ produces a gradient of cell deformation which changes with the lift, the same gradient $g$ arises near the resting Earth. In one case the gradient is produced by the propellant, in the other case by the mass "body" of the Earth.

The simplest concept of space structure is given by honeycomb there one can see direct lines and planes. But, 
as a particle is formed, the outer cells are deformed forming not planes but curved surfaces which coat the particles like onion layers. The whole complex of these curved surfaces can be referred to as three-dimensional space curvature, and to see this curvature we needn't enter the fourth measurement. Deformed space cells practically visualize the curvature introduced by Einstein into the theory of gravity.

The structure of outer layers manifests itself most vividly in case of a heavy particle if it exists - a planceon with its mass $m^{*}$ consisting just of one cell:

$$
m^{*}=\sqrt{\frac{\hbar c}{2 G}} \cong 1,6 \cdot 10^{-5} \mathrm{gr}
$$

The number of cells in the subsequent layers coating the particle grows as $N \sim r^{2}$, so with an increase in the distance from the particle the deformation of space cells decreases constantly. (This is, however, not just a monotonous decrease but a striking physical effect, that is, collectivization or clusterization of cells in certain layers). Every layer is a two-dimensional surface, so the deformation of one cell will change to the deformation of a cluster containing already four cells. The numbers 2 and 3 of a cell are excluded because granular space is uniform and isotropic since it is composed of similar cells. The minimum distance at which four cells become a cluster is $r_{1}=4 r_{0}$ because this spherical layer can be coated with clusters consisting of four cells. The next cluster contains nine cells, and distance to the second spherical layer increases up to $r_{0}=9 r_{0}$, and so on. We can observe almost visually the formation of gravitational energy levels or, in other words, the quantization of a gravitational field. The quantization of the Coulombian field in a hydrogen atom was considered by us in another article.

The same dependence of Coulombian and gravity forces on distance means that their energy levels are formed at the same distances from the proton. But if the first gravitational level in a hydrogen atom begins with one elementary cell, an electric cluster has $4,17 * 10^{42}$ cells.

The granular structure of space enables us to answer numerous questions where Bohr's model and quantum mechanics are at a deadlock:

What is an energy level?

Why is an electron retarded on a level?

Why is half the energy of an electron released as it passes from one level to another one?

What is the motion of an electron between levels: smooth or stepwise?

Why doesn't an electron throw off a photon on the ground level which means that the atom is table?

What is time asymmetry?
A fundamental characteristic of a gravitating body is the square of escape velocity at the distance $r$ from the body rather than of circular velocity. Lorentz's transformations acquire their real physical meaning as an instrument of account for the maximum deformation of cells in a given reference system. These transformations can be called as generalized Lorentz's transformations. To calculate correctly physical processes we should substitute the maximum value $\left(v^{2} / c^{2}\right)_{\max }$ into the well-known root $\sqrt{1-v^{2} / c^{2}}$ taking into account not only the velocity of an object in a given reference system but also the escape velocity of the massive body within the boundaries of which a physical effect is considered. It should be noted that the square of escape velocity is equal to the twice potential of gravitational field:

$$
v_{2}^{2}=\frac{2 G M}{r}=2 \varphi .
$$

Schwarzschield's solution is only related to a gravitational field whereas the metrics found by us with the use of generalized Lorentz's transformations can be observed in any field of forces if only it had a spherical symmetry and the basis had only radial velocities.

The first attempts, including those of Einstein, to apply Lorentz's transformations to studies of gravitation failed because the nature of "the two" remained a mystery that could be revealed just by turning to the material granular structure of space.

Besides, in the granular space theory the expression:

$$
r_{g} / r=\frac{2 G M}{r c^{2}}=\frac{v_{2}^{2}}{c^{2}},
$$

has quite a different physical meaning.

As it has been said above, $v_{2}$ here is similar to the escape velocity and characterizes the real deformation of space cells at the distance $r$ from the massive body M.

The fact that the true meaning of $r_{g} / r$ has not been known so far enables us to make a surprising conclusion that in the Schwarzschield's solution contains a singular sphere where the meanings of $\mathrm{d} x^{\circ}$ and $\mathrm{d} r$ vary.

Physicists have so far been trying to solve the problem of quantum gravitation development which is needed to explain physical processes occurying in singularity.

In our case there is no singularity at all. It can exist in Schwarzschield's metrics in the limit with $r=r_{g}$ which is unattainable for a real body since in this case $\beta=v_{2} / c=$ 1, i.e. $v_{2}=c$ !

Even in his own reference system the outer observer can never get the gravitational radius $r_{g}$. The statement that we are living inside a huge black hole is rather intriguing since the gravitational field potential at the boundary line of the Universe reaches the squared velocity of light: $\varphi \simeq c^{2}$ and, hence, all physical processes 
slow down to zero. But we don't feel uncomfortable at all.

This, at last, enables us to get rid of all the difficulties relating to singularity both for a massive body and for the Universe on the whole. In more detail we shall consider some problems dealing with cosmology in a separate article.

It is of great interest to look back into history. Geisenberg was the first to formulate the foundations of quantum mechanics - matrix mechanics. Short time later Shrödinger found his famous wave equation. But most of the physicists, including Dirack, preferred working with matrix quantum mechanics. Shrödinger had nothing to do but to show that the two formulations of quantum mechanics were equivalent which he performed with success.

We have to solve a similar problem: as the results of Einstein's theory of gravity and the theory of granular space coincide, we must find points of their contact. Partially we already did it when we found out that the formation of any particle is responsible for the fact that the former spatial planes consisting of cells form, as they turn to curved surfaces, a laminated structure around the particle. The curved surfaces of individual layers permit using curved (spherical) coordinates, the third coordinate makes it possible to account for the change in cell deformation depending on the distance to the particle and, finally, the fourth coordinate, the time coordinate, accounts for the deceleration of the processes during the deformation of the cells surrounding the particle. This deceleration has so far been a striking physical effect, though quite incomprehensible. And only in studying the real material structure of space we can get a round grasp of this phenomenon. It is rather puzzling that nobody has ever observed the acceleration of physical processes.

This problem can be easily solved by observing a moving particle in an imaginary experiment. In our article "Weak Interaction and the Nature of Virtual Particles" [10], we determined the wall thickness of an elementary space cell $-\Delta l \simeq 10^{-100} \mathrm{~cm}$. It is quite probable that the mass carried by kinetic energy fills the cells surrounding the particle, the walls become thicker.

This excess matter reduces the elasticity of elementary cells surrounding a massive body which, in its turn, causes the velocity of light to drop. The radial velocity of a light signal is equal to $C_{r}$ when the time is counted by a far-remote clock:

$$
C_{r}=\frac{\mathrm{d} r}{\mathrm{~d} t}=c\left(1-\frac{r_{g}}{r}\right)=c\left(1-\frac{v_{2}^{2}}{c^{2}}\right) .
$$

It should be noted that empty space, only due to its curvature, cannot help either slowing down the course of physical process or stopping light. In this case material space is needed.

The theory of granular space, like Einstein's gravity theory, predicts that a rotating body sets up around itself both a static (potential) field and a stationary rotational field that looks like the stationary magnetic field of a rotating charged body due. Its rotational character is caused by the torsion deformation of the space cells around the body.

It is evident that in this case the acceleration of physical processes is completely excluded.

We have just resolved into components such a composite object as the curvature of four-dimensional space-time used in Einstein's theory. All this enables us to state that Einstein's equation works perfectly in tree-dimensional material space by describing the deformation (curvature) of cell clusters formed by massive bodies and thus ruling out the idea of void curvature. In our further work we shall consider in more detail the mechanisms of both gravitational and Coulomb interactions. Here we want just to note that the attraction of bodies occurs when the deformation of cells beyond the bodies is larger than between them and their repulsion is characterized by a high deformation of the cells between the bodies. The difference in cell deformation sets up a deformation gradient referred to as force. The former straight lines formed by the cells as a result of their deformation become curved and the planes turn to curved surfaces which form the basis for space curvature mentioned by H. Lobachevsky, J. Bolyai, G. Riemann, Gauss and Einstein.

It is well-known that Einstein's theory of gravity has not any gravitational field at all, it only contains space-time curvature formed by moving masses. The theory of granular space states that it is only material space that can be characterized by curvature. An "empty" geometry lacks both mass, energy and momentum. Thus, space curvature becomes a secondary effect, while the deformation of material space cells acts as "first violin".

All the attempts to quantize directly Einstein's theory have failed because of the presumed tensor nature of the gravitational field and due to interaction nonlinearity.

The essence of nonlinearity consists in the fact that gravitational field possesses potential energy and, hence, mass since $E=m c^{2}$. The conclusion, that this mass is a source of gravity by itself, is mistaken. This additional gravity field, in its turn, acts as an additional source of gravity and so an. As a result, a peculiar "matreshka" made up of field sources is formed which gives rise to nonlinearity.

For counterexample it should be noted that a Coulomb field, too, possesses potential energy and, hence, mass but it cannot form any additional gravity field and remains linear. 
According to the theory of granular space, the essence of any physical field is deformed apace cells around a material body (a particle). Being elements of a gravitational field these particles do not set up any additional field. A prominent example of gravitational field linearity for the time being is the same distance dependence of both Coulomb and gravitational fields.

Consequently, Schrödinger equation is suitable for both the fields and predicts the existence of both gravitational and Coulomb levels.

In our work «Gravitational Energy Levels and the Problem of Microwave Radiation of the Universe» [11] we could find that it is gravitational energy levels that form quasi- black-body radiation with $T \simeq 2.7 \mathrm{~K}$ around the Earth but neither WMAP nor PLANK have yet measured the absolute flaxes of this radiation away from the Earth in order to be sure of its terrestrial origin rather than to remove the term «relict». We need just one figure - the quantity of absolute intensity of cosmic microwave background radiation in the millimeter range? Measured far away from the Earth to study the problem of "relict" radiation and graviton. This measurement should have been done even forty years ago.

Gravitational energy levels are of great importance for cosmology. Only owing to them, particles get together forming stars and planets just as electrons are arranged on the energy levels formed by nuclei.

\section{Mechanism of Gravitational and Coulombian Interactions}

Not let us unravel the gravity mechanism by studying in an imaginary experiment two massive bodies initially resting in a system of fixed stars. Each body a laminated structure of deformed space cells around itself. The layers of space cells between the bodies are opposite in curvature, their actions on each other weaken the total curvature, i.e. decrease the deformation of the cells in inner space. The curvatures of the layers behind the bodies coincide increasing the total deformation. Thus, a difference in cell deformation in front of and behind the bodies is set up - the potential gradient $\varphi$ only remains for us to state that the bodies begin inevitably to move to meet each other (Figure 4):

The bodies are not attracted together but it is space that brings them together due to the cell deformation gradient. Huygens was wrong when he blamed Newton for his law of gravitation because the latter had not unraveled the mechanism of gravitation. As Newton anticipated, the mechanism of gravitation would be cleared up only 350 years later.

The substance of the destructed cells behind the body, whose deformation is larger, is forced out into the front

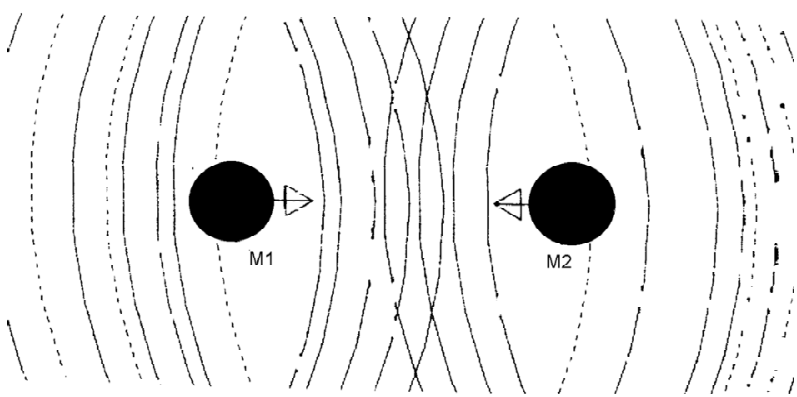

Figure 4. The simplest mechanism of gravity.

semisphere where the deformed cells are subjected to destructive interference. This process demonstrates real material transfer of potential energy to kinetic one. The source of potential energy has already been considered. Space in this situation acts as a creditor and not as a money-lender because it does not take any interest from the debtor. As these bodies collide, part of the substance of kinetic energy called the bond energy will be released and, if we try to pall them apart at the former distance, we have to return the same amount of substance that was released, to the space.

If the particles have electric charges, the substance transferred to the by space is a lot of times larger than the substance imparted to a neutral particle by gravitation. The mechanism of Coulombian interaction very much resembles that of gravitational interaction which results in the same dependence of these forces on the charges and the distance between them.

The positive and negative curvatures of the clusters on the surfaces of a position and electron demonstrate a unique possibility of space either to pull together particles (attraction) or to repel them from each other (repulsion).

Over two thousand years ago people were looking for the cause of gravity offering various hypotheses, but only the research into the real structure of space has shown how deep the mystery of gravitation was hidden: the difference in cell deformation in front of and behind the body creates a gravitational force. The exchange mechanism offered by W.Gilbert, a court physician of Elizabeth, queen of England, turns out to be groundless. Only as two particles collide, energy and momentum can be transferred from the shell-particle to the target-particle, and here we can speak of exchange interaction but the mathematical description of a collisional process, as opposed to the static one, becomes so much more difficult that it is beyond the worst expectations. Indeed, in a collisional process the deformation of a huge number of cells, $\sim 10^{60}$, assumes the most fantastic forms.

The maximum deformation of cells is an objective property and is independent of the reference system in use. But it is velocity of light that is hidden behind it. 
This foot makes the velocity of light constant in any inertial system far away from gravitating bodies as well as independent of the motion of the source and the observer.

However the velocity of light measured in different reference system will differ according to the direction (along and against) of motion as it has been said above.

This also makes it impossible to convey information at a rate higher than the velocity of light. The gradient mechanism of both gravitational and Coulombian interactions removes, as last, the antagonism between shortrange and long-range interactions: the force arising on the particle surface is transferred from cell to cell at a long distance smoothly realizing far-range interaction. For example, the force acting on a planet is the sum of forces acting on every elementary particle of this planet.

The local nature of force unravels the enigma of origin of such forces as centrifugal, Coriolis and the force suspending a gyroscope and preventing it from falling on the Earth. These forces have so far been considered fictitious since we could not point out to a body which would act on the object under consideration. Later on we are going to consider in detail the action of these forces but now we want to note that any attempt to understand these phenomena in purely a geometrical way is ungrounded. Guided by the idea "everything from geometry" Einstein interchanged cause and effect: cause-the deformation of elementary space cells, effect - the change of the arrangement of cells and their physical properties, that is, what is called now as the curvature of four-dimensional diversity of space-time.

In Einstein's theory of gravity the linear element becomes a generalized quadratic form:

$$
\mathrm{d} s^{2}=g_{\mu \nu} \mathrm{d} x^{\mu} \mathrm{d} x^{v} .
$$

In the description of gravitational field the metric tensor $g_{\mu v}$ takes the central place. According to Einstein, these values act as "gravitational potentials". The number of "independent potentials" in the general case is 10 because $g_{\mu \nu}=g_{v \mu}$. In gravitational fields of individual heavenly bodies, stars and planets for instance, a reference system can be usually chosen so that the quantity $g_{00}$, that is, the coefficient before $\mathrm{d} t^{2}$, would be the most essential. In Schwarzschield's solution considered above:

$$
g_{00}=\left(1-\frac{2 \varphi}{c^{2}}\right)=\left(1-\frac{2 v^{2}}{c^{2}}\right) .
$$

This quantity cannot act as a gravitational potential since it is dimensionless. As far back as 50 years ago Zeldovich was right to state that the tensors $R_{i k}$ and $R$ appearing in Einstein's equation of gravity describe the elasticity of space and the component $g_{00}$ suggests clearly that the quantity $\varepsilon=v^{2} / c^{2}$ characterizes the relative de- formation of elementary space cells that plays a dominant role in the theory of granular space.

As Newton thought, free fall occurs due to the gravitational force. Moreover, all the gravitational effects in the solar system (Mercury's perihelion displacement, light deflection by the Sun, radio signal lag, hyroscope precession) are caused by gravity force. The mechanism of gravitational interaction under consideration completely rules out a gravitational shadow and, on the contrary, the Earth's attraction by the Sun increases when the Moon is placed between them.

\section{Conclusions}

Fifty years ago Fock reasoned that "generally speaking, there is not any relativity in the general relativity theory" [12]. These words were dictated by Fock's wish to clarify Einstein's theory of gravity by getting rid of meaningless general relativity in it.

The theory of granular space extends farther: untangling the physical meaning of velocity, introducing generalized Lorentz's transformations and proving, in the general case, the absence of process - invariance with form-invariance of all physical laws-shows that the Universe does not know the concept of relativity. Only mind makes use of different reference system to simplify the procedure of obtaining quantitative results, but Space performs all its calculations in one reference systemabsolute.

At present many physicists create an extremely complicated and speculative inflationary hypothesis [13].

A study into the real structure of space is able even now to give us, at least, a rough idea of the processes of attraction and repulsion and even to observe the mechanisms of all physical interactions. We shall go on studying the real structure of space in our next work.

\section{References}

[1] M. Planck, "The Unity of the Physical Patter of the World," Nauka, Moscow, 1996.

[2] V. Konushko, "Concepts of Granular Space Theory," Sputnik, Moscow, 1999.

[3] R. Feynman, "The Feynman Lectures on Physics," Addison-Wesley Publishing Company, London, 1963.

[4] V. Konushko, "Granular Space and the Problem of Large Numbers," International Journal of Modern Physics, 2011 (In Press).

[5] J. Wheeler, "Einsteins Vision," Springer Verlag, New York, 1968.

[6] T. Jacobson and R. Parentani, "The Echo of the Black Holes," Scientific American, No. 3, 2006, p. 17. 
[7] A. Smolin, "Atom's Space and Time," Scientific American, No. 4, 2004, p. 20.

[8] H. Bondi, "Assumption and Myth in Physical Theory," Cambridge, 1967.

[9] V. Braginsk and A. Polnarev, "Surprising Universe," Nauka, Moscow, 1985.

[10] V. Konushko, "Weak Interaction and the Nature of Virtual Particles," International Journal of Modern Physics,
No. 4, 2011, pp. 45-57.

[11] V. Konushko, "Gravitational Enerdy Levels and the Problem of Microwave Radiational of the Universe," International Journal of Modern Physics, 2011 (In Press).

[12] V. Fock, "The Theory of Space Time and Gravitation," Pergamon Press, London, 1959.

[13] A. Levin, "Trillion Years before Big Bang," Popular Mecanics, No. 6, 2010, pp. 47-50. 